\title{
SCALABLE NEWTON-KRYLOV-BDDC AND FETI-DP DELUXE SOLVERS FOR DECOUPLED CARDIAC REACTION-DIFFUSION MODELS
}

\author{
NGOC MAI MONICA HUYNH ${ }^{1}$, LUCA F. PAVARINO ${ }^{1}$ AND SIMONE SCACCHI ${ }^{2}$ \\ ${ }^{1}$ Dipartimento di Matematica, Università degli Studi di Pavia \\ via Ferrata 5, 27100 Pavia - Italy \\ ngocmaimonica.huynh01@universitadipavia.it, luca.pavarino@unipv.it \\ ${ }^{2}$ Dipartimento di Matematica, Università degli Studi di Milano \\ via Saldini 50, 20133 Milano - Italy \\ simone.scacchi@unimi.it
}

Key words: Domain decomposition, FETI-DP and BDDC preconditioners, deluxe scaling, Bidomain system, high-performance computing.

\begin{abstract}
Two parallel Newton-Krylov Balancing Domain Decomposition by Constraints (BDDC) and Dual-Primal Finite Element Tearing and Interconnecting (FETI-DP) solvers are analyzed and numerically studied for implicit time discretizations of the Bidomain equations. This system models the cardiac bioelectrical activity and it consists of a degenerate system of two non-linear reaction-diffusion partial differential equations (PDEs), coupled with a stiff system of ordinary differential equations (ODEs). A non-linear algebraic system arises from a finite element discretization in space and an implicit discretization in time, based on decoupling the PDEs from the ODEs. Within each Newton iteration, the Jacobian linear system is solved by a Krylov method, accelerated by BDDC or FETI-DP preconditioners, both augmented with the recently introduced deluxe scaling of the dual variables. Several parallel numerical tests on Linux clusters confirm a novel polylogarithmic convergence rate bound, showing scalability and quasi-optimality of the proposed solvers.
\end{abstract}

\section{INTRODUCTION}

The aim of this work is to present some recent developments of Newton-Krylov solvers for implicit time discretizations of the Bidomain model, preconditioned by Balancing Domain Decomposition by Constraints (BDDC) and Dual-Primal Finite Element Tearing and Interconnecting (FETI-DP) algorithms. The Bidomain model describes the propagation of the electric signal in cardiac tissue by means of a degenerate parabolic system of two non-linear reaction-diffusion partial differential equations (PDEs), modelling the evolution of the transmembrane electric potential $[5,17,18,19]$. The PDEs are coupled through the non-linear reaction term with a system of ordinary differential equations (ODEs), known as membrane model, describing the ionic currents flowing inward and outward the cell membrane and the dynamics of the associated gating variables.

So far, operator splitting strategies [2, 22] or semi-implicit time discretizations [3, 25] have been widely preferred to fully implicit ones [15], as the latter are computationally very expensive if the electrical 
model is coupled with very stiff and high-dimensional non-linear system of ODEs $[12,23]$. In the same fashion as in previous works of some of the Authors [13, 14, 21] (where overlapping Schwarz preconditioners were considered) we propose here a solution approach based on the decoupling of the two models: the ODEs system is solved first, then the non-linear problem arising from the implicit time discretization of the Bidomain system is solved and updated. The preconditioners taken into account are FETI-DP and BDDC dual-primal preconditioners.

FETI-DP methods were proposed in Ref. [8], while BDDC preconditioners were introduced in Ref. [6]. Thus far, BDDC algorithms have been applied to semi-implicit time discretizations of the Bidomain model (see e.g. [25]), while Newton-Krylov-BDDC solvers have been developed for the non-linear system arising from the discretization of finite elasticity equations modelling the mechanical contraction and relaxation of the cardiac muscle [3, 16].

As the two preconditioners have been proven to be spectrally equivalent [11], we provide a theoretical estimate for the condition number bound of the preconditioned operator, based on the deluxe-scaling recently introduced in [7]. Parallel numerical tests on an idealized left ventricle confirm our bound and show robustness and computational equivalence between the proposed dual-primal substructuring algorithms, thus encouraging further investigations with realistic geometries and the development of a monolithic solver for the electro-mechanical model.

\section{CARDIAC ELECTRICAL MODELS}

We study here the Bidomain system [17], which models the propagation of the electrical signal in the

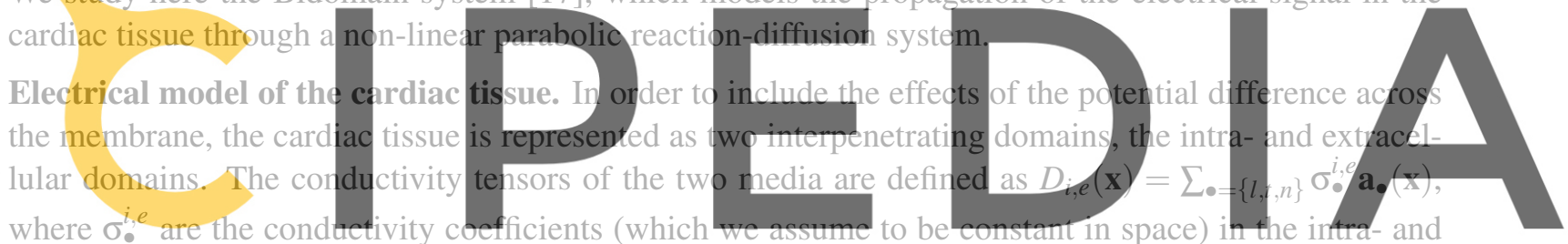

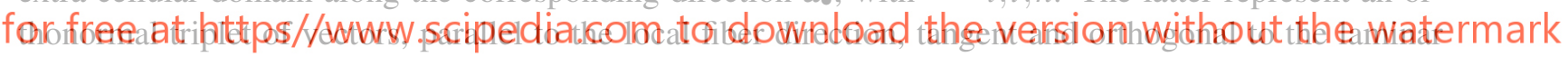
sheets, respectively, defined at each point of the cardiac domain. This construction allows us to represent the arrangement of cardiac cells in fibers set as laminar sheets running counterclockwise from the epicardium to the endocardium [9].

Defining the intra- and extracellular electric potentials $u_{i, e}: \Omega \times(0, T) \rightarrow \mathbb{R}$, the transmembrane potential $v=u_{i}-u_{e}$ and the gating and ionic concentration variables $w: \Omega \times(0, T) \rightarrow \mathbb{R}^{N_{w}}$, being $\Omega$ the cardiac domain, then the parabolic-parabolic formulation of the Bidomain system reads:

$$
\begin{cases}\chi C_{m} \frac{\partial v}{\partial t}-\operatorname{div}\left(D_{i} \cdot \nabla u_{i}\right)+I_{\text {ion }}(v, w)=0 & \text { in } \Omega \times(0, T), \\ -\chi C_{m} \frac{\partial v}{\partial t}-\operatorname{div}\left(D_{e} \cdot \nabla u_{e}\right)-I_{\text {ion }}(v, w)=-I_{\text {app }}^{e} & \text { in } \Omega \times(0, T), \\ \frac{\partial w}{\partial t}-R(v, w)=0, & \text { in } \Omega \times(0, T), \\ v(x, t)=u_{i}(x, t)-u_{e}(x, t) & \text { in } \Omega \times(0, T),\end{cases}
$$

where $I_{\text {app }}^{e}: \Omega \times(0, T) \rightarrow \mathbb{R}$ is the extracellular applied current, needed for the potential to start propagating, with appropriate initial values and boundary conditions. Moreover, in order to guarantee well 
posedness, the compatibility condition $\int_{\Omega} I_{\text {app }}^{e} d x=0$ must hold.

Results on existence, uniqueness and regularity of the solution of (1) have been extensively studied, see for example [4].

Ionic model of cell membrane. The equations describing the propagation of the electrical signal are usually coupled through the reaction term to a system of Ordinary Differential Equations (ODEs) which describes the ionic currents flowing inward and outward the cell membrane. Here we consider the phenomenological Roger-McCulloch ionic model [20], which presents only one gating variable. In this case, $I_{\text {ion }}(v, w)$ is given by

$$
I_{\text {ion }}(v, w)=G v\left(1-\frac{v}{v_{t h}}\right)\left(1-\frac{v}{v_{p}}\right)+\eta_{1} v w,
$$

while the equation for the gating variable reads

$$
R(v, w)=\eta_{2}\left(\frac{v}{v_{p}}-w\right)
$$

where $G, v_{t h}, v_{p}, \eta_{1}$ and $\eta_{2}$ are constant coefficients.

3 NUMERICAL METHODS

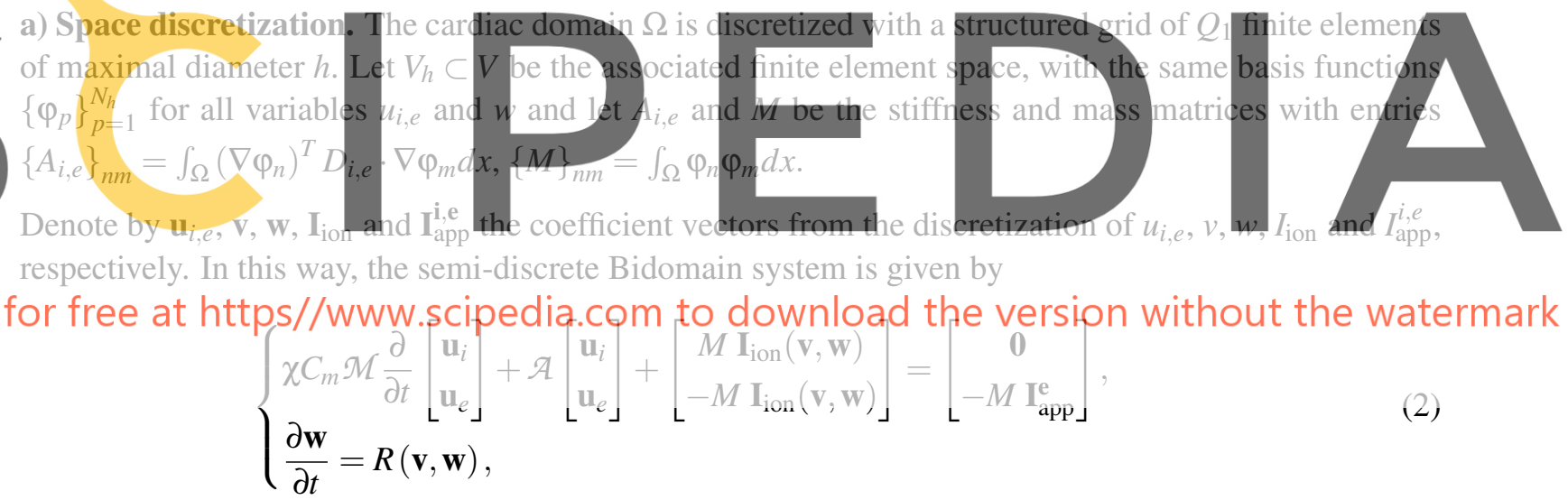

where $\mathcal{A}$ and $\mathcal{M}$ are the stiffness and mass block-matrices $\mathcal{A}=\left[\begin{array}{cc}A_{i} & 0 \\ 0 & A_{e}\end{array}\right], \quad \mathcal{M}=\left[\begin{array}{cc}M & -M \\ -M & M\end{array}\right]$.

b) Decoupled implicit time discretization. Realistic ionic models are very complex and can present up to fifty non-linear ODEs (see e.g. [12,23]). This makes the solution of the non-linear problems arising from fully implicit time discretizations of the Bidomain system coupled with these systems computationally very expensive.

In the literature, common alternatives consider implicit-explicit (IMEX) schemes and/or operator splitting, where the diffusion terms are treated separately from the reaction $[2,25,22]$.

The strategy we adopt here is to decouple the gating variable $w$ from the intra- and extracellular potentials $u_{i}$ and $u_{e}$ as in [13]. At each time step, this decoupled Backward Euler approach consists in: 
- Step 1: update gating and ionic variables. Given $\mathbf{u}_{i, e}^{n}$ (hence $\mathbf{v}^{n}$ ) at the previous time step $t_{n}$, compute $\mathbf{w}^{n+1}$ by solving the membrane model

$$
\mathbf{w}^{n+1}-\tau \mathbf{R}\left(\mathbf{v}^{n}, \mathbf{w}^{n}\right)=\mathbf{w}^{n},
$$

where $\tau=t_{n+1}-t_{n}$ is the current time step.

- Step 2: solve the Bidomain system. Given $\mathbf{u}_{i, e}^{n}$ at the previous time step and given $\mathbf{w}^{n+1}$, compute $\mathbf{u}^{n+1}=\left(\mathbf{u}_{i}^{n+1}, \mathbf{u}_{e}^{n+1}\right)$ by solving the non-linear system $\mathbf{F}\left(\mathbf{u}^{n+1}\right)=\mathbf{G}$ derived from the Backward Euler scheme applied to the Bidomain equations, with

$$
\mathbf{F}\left(\mathbf{u}^{n+1}\right)=\left(\chi C_{m} \mathcal{M}+\tau \mathcal{A}\right)\left[\begin{array}{l}
\mathbf{u}_{i}^{n+1} \\
\mathbf{u}_{e}^{n+1}
\end{array}\right]+\tau\left[\begin{array}{c}
M \mathbf{I}_{\text {ion }}\left(\mathbf{v}^{n+1}, \mathbf{w}^{n+1}\right) \\
-M \mathbf{I}_{\text {ion }}\left(\mathbf{v}^{n+1}, \mathbf{w}^{n+1}\right)
\end{array}\right], \quad \mathbf{G}=\chi C_{m} \mathcal{M}\left[\begin{array}{l}
\mathbf{u}_{i}^{n} \\
\mathbf{u}_{e}^{n}
\end{array}\right]+\tau\left[\begin{array}{c}
\mathbf{0} \\
-M \mathbf{I}_{\mathrm{app}}^{\mathbf{e}}
\end{array}\right] .
$$

\subsection{Dual-primal preconditioners for Newton-Krylov solvers}

The strategy we propose for the solution of the non-linear system in Step 2 consists in a Newton-Krylov method, as in Ref. [13, 14, 21]: a Newton scheme is applied as outer iteration and the Jacobian linear system arising at each Newton step is solved by a Krylov method preconditioned by a dual-primal iterative substructuring algorithm.

Newton scheme. The outer Newton iteration can be summarized as

choose an initial value $\mathbf{u}^{0}=\left(\mathbf{u}_{i}^{0}, \mathbf{u}_{e}^{0}\right)$;
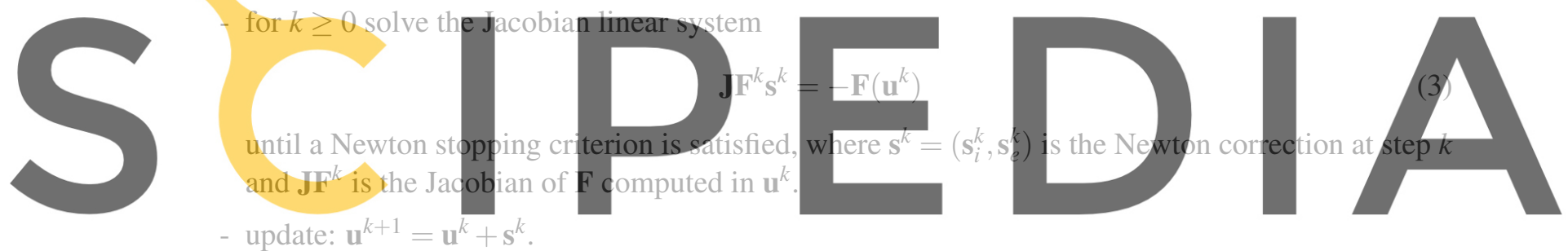

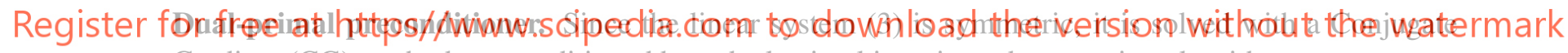
Gradient (CG) method, preconditioned by a dual-primal iterative substructuring algorithm.

Let $\left\{\Omega_{i}\right\}, i=1, \ldots, N$, be a decomposition of the cardiac domain $\Omega$, into non-overiapping subdomains of diameter $H_{i}$, such that $\bar{\Omega}=\cup_{i=1}^{N} \bar{\Omega}_{i}, \Omega_{i} \cap \Omega_{j}=\emptyset$ if $i \neq j$ and let $H=\max _{i} H_{i}$ be the maximum diameter while denote with $\Gamma=\cup_{i=1}^{N} \partial \Omega_{i} \backslash \partial \Omega$ the interface (for further details, see Ref. [24]).

As in classical iterative substructuring, we implicitly eliminate the degrees of freedom interior to each subdomain, thus reducing the problem to one on the interface $\Gamma$. The resulting Schur complement system

$$
S_{\Gamma} \mathbf{x}_{\Gamma}=\mathbf{g}_{\Gamma},
$$

is then solved with a preconditioned Krylov method.

In this work, we focus on the most common dual-primal iterative substructuring algorithms, BDDC and FETI-DP.

FETI-DP methods were first proposed in Ref. [8] and are based on transposing from the linear system to a constrained minimization problem, where iterations are made over the additional Lagrange multipliers introduced in the associated saddle point formulation. 
Conversely, BDDC methods, introduced in Ref. [6] as an alternative to FETI-DP, provide a preconditioner for the discretized linear problem (4) and can be viewed as an Additive Schwarz method of Neumann-Neumann type [10].

As it has been proven that these two algorithms are spectrally equivalent [11], it is possible to derive the following convergence rate estimate for the preconditioned operator, which holds for both in case the same coarse space is chosen. In particular, the novelty of this bound consists in the application of the deluxe scaling, recently introduced in Ref. [7]. We observe that in this application the condition number is independent from the number of subdomains, as in most of the convergence bounds for FETI-DP and BDDC operators.

Theorem 3.1 If the deluxe scaling is used, the condition number of the FETI-DP and BDDC preconditioned Bidomain operators satisfy

$$
\text { cond }\left(P^{-1} Q\right) \leq \max _{\substack{k=1, \ldots, N \\ \star=\ldots, \rho}} \frac{\tau \sigma_{M}^{\star(k)}+H^{2}\left(\chi C_{m}+\tau K_{M}\right)}{\tau \sigma_{m}^{\star(k)}}\left(1+\log \left(\frac{H}{h}\right)\right)^{3},
$$

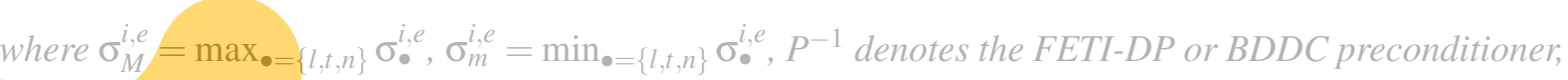
$Q$ is the Bidomain Jacobian (Schur complement saddle point system), and $K_{M}$ is a constant independent of the subdomain diameter $H$ and mesh size $h$.

4 NUMERICAL RESULTS
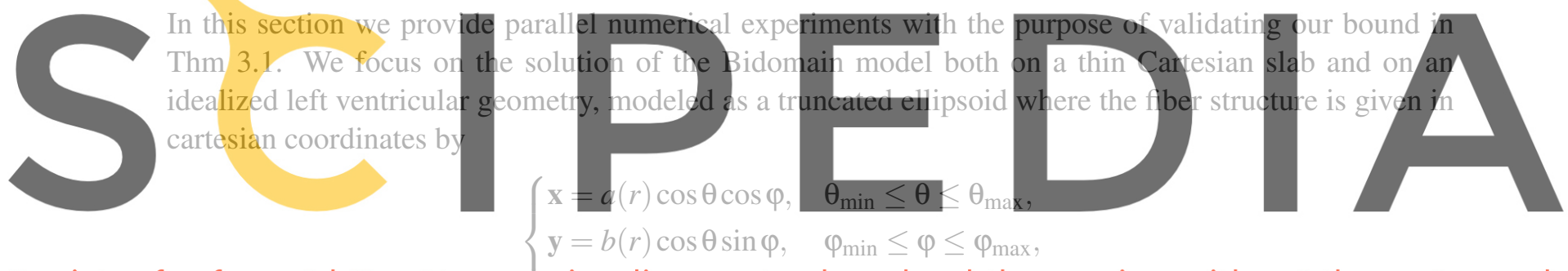

$\varphi_{\min } \leq \varphi \leq \varphi_{\max }$.

\section{Register for free at https//www.scipe(d)iaicom to dołwnłoad the version without the watermark}

where $a(r)=a_{1}+r\left(a_{2}-a_{1}\right), b(r)=b_{1}+r\left(b_{2}-b_{1}\right)$ and $c(r)=c_{1}+r\left(c_{2}-c_{1}\right)$ with $a_{1,2}, b_{1,2}$ and $c_{1,2}$ coefficients of the main axes of the ellipsoid.

The weak scaling tests are performed on the supercomputer Galileo from Cineca centre, while the strong scaling tests are computed on the Linux cluster Eos at the University of Pavia; the experiments over an heartbeat time interval are carried out on the Indaco cluster from the University of Milan. Our C code is based on the PETSc library [1] from Argonne National Laboratory.

The outer Newton loop is solved with the non-linear solver SNES within PETSc library, which implements a Newton method with cubic backtracking linesearch. The linear system arising from the discretization of the Jacobian problem at each Newton step is solved with a Conjugate Gradient (CG) method, preconditioned by BDDC or FETI-DP preconditioners (from the PETSc library) and, for the last set of experiments, the Boomer Algebraic MultiGrid (bAMG, from the Hypre library).

We compare the following quantities: the average Newton iterations per time step nit, the average conjugate gradient iterations per Newton iteration lit and the average CPU solution time per time step in seconds time. Unless otherwise specified, the tests run for 40 time steps over the time interval $[0,2] \mathrm{ms}$. 
Test 1: weak scaling. We report first a weak scaling test on both slab and ellipsoidal domain. For both geometries, we fix the local mesh size to $16 \cdot 16 \cdot 16$ and we increase the number of subdomains from 32 to 2048, thus resulting in an increasing slab geometry and in an increasing portion of ellipsoid. From Table 1, it is evident the good performance of the dual-primal algorithms: the average number of linear iterations per Newton iteration lit is low and does not increase with the number of subdomains, except for BDDC for the increasing slab.

Table 1: Slab (top table) and ellipsoidal (bottom table) domains. Weak scaling test on the cluster Galileo over time interval of $[0,2] \mathrm{ms}, 40$ time steps. Increasing number of subdomains from 32 to 2048 and fixed local mesh size of $16 \cdot 16 \cdot 16$ elements.

\begin{tabular}{|r|c|r|r|r|r|r|r|}
\hline \multicolumn{10}{|c|}{ Slab } \\
\hline \multirow{2}{*}{ procs } & \multirow{2}{*}{ dofs } & \multicolumn{3}{|c|}{ BDDC } & \multicolumn{3}{|c|}{ FETI-DP } \\
\cline { 3 - 9 } & & nit & lit & time & nit & lit & time \\
\hline 32 & 278,850 & 1 & 22 & 6.1 & 1 & 10 & 6.0 \\
64 & 553,410 & 1 & 27 & 6.2 & 1 & 11 & 6.0 \\
128 & $1,098,306$ & 1 & 32 & 7.6 & 1 & 10 & 7.4 \\
256 & $2,188,098$ & 1 & 39 & 7.2 & 1 & 10 & 7.9 \\
512 & $4,359,234$ & 1 & 48 & 10.1 & 1 & 10 & 11.1 \\
1024 & $8,701,506$ & 1 & 63 & 13.8 & 1 & 10 & 18.7 \\
2048 & $17,369,154$ & 1 & 78 & 33.5 & 1 & 10 & 63.2 \\
\hline
\end{tabular}
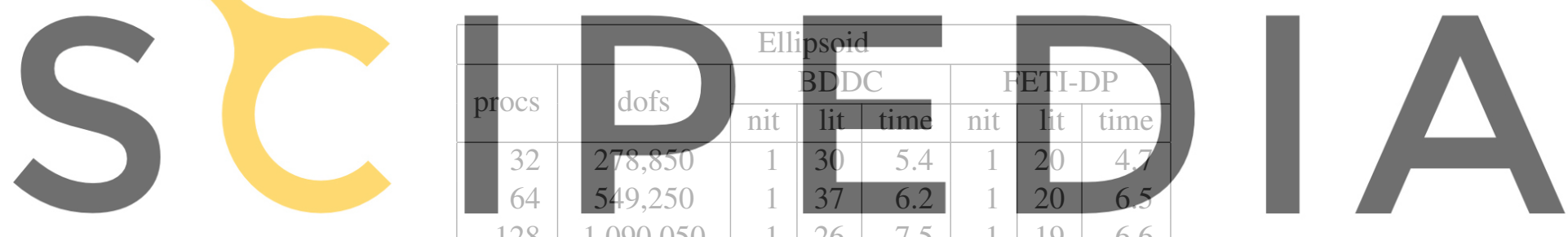

Register for free at https//w
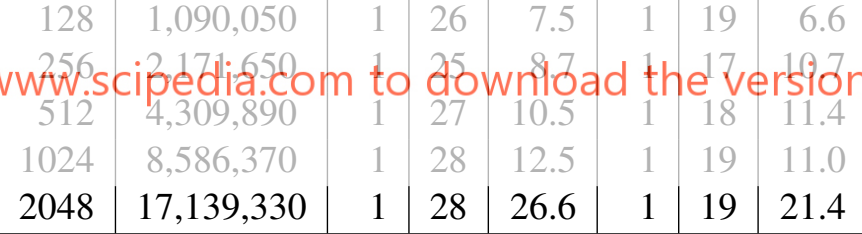

Test 2: strong scaling. We compare the performance of BDDC and FETI-DP preconditioners while varying the number of processors from 64 to 256 over a time interval of $[0,100] \mathrm{ms}$, for a total of 2000 uniform time steps. The global mesh is fixed to $192 \cdot 96 \cdot 24$ elements $(936,050 \mathrm{dofs})$.

For the ellipsoid, we observe a reduction of the CPU time while increasing the number of subdomains (Table 2), although the average number of linear iterations does not decrease as expected (Figures 1 and 2). The geometry has a strong effect, since with the slab domain the things scale much better.

Test 3: whole heartbeat simulations. In this last set of tests, we compare the performance of our dualprimal and the multigrid preconditioners during a whole heartbeat. We fix the number of subdomains to $128=8 \cdot 8 \cdot 2$ and the global mesh size to $192 \cdot 96 \cdot 24$, obtaining local problems with 8,450 dofs. We consider a time interval of $[0,170] \mathrm{ms}$ for a total of 3400 time steps for a portion of ellipsoid defined by $\varphi_{\min }=-\pi / 2, \varphi_{\max }=0, \theta_{\min }=-3 / 8 \pi$ and $\theta_{\max }=\pi / 8$, while on the slab of dimensions $1.92 \times 1.92 \times$ 
Table 2: Strong scaling test over a time interval of $[0,100] \mathrm{ms}$ (2000 time steps) on Eos cluster. Fixed global mesh size of $192 \cdot 96 \cdot 24$ elements, increasing number of processors from 64 to 256 .

\begin{tabular}{|c|c|c|c|c|c|c|c|}
\hline \multirow{2}{*}{} & \multirow{2}{*}{ procs } & \multicolumn{3}{|c|}{ BDDC } & \multicolumn{3}{c|}{ FETI-DP } \\
\cline { 3 - 8 } & & nlit & lit & time & nlit & lit & time \\
\hline \multirow{4}{*}{ ellipsoid } & 64 & 2 & 42 & 21.36 & 2 & 30 & 20.85 \\
& 128 & 2 & 32 & 8.61 & 2 & 23 & 8.17 \\
& 256 & 2 & 42 & 9.34 & 1 & 68 & 13.02 \\
\hline \multirow{4}{*}{ slab } & 64 & 1 & 19 & 16.23 & 1 & 12 & 18.48 \\
& 128 & 1 & 19 & 9.52 & 1 & 12 & 6.18 \\
& 256 & 1 & 17 & 4.97 & 1 & 11 & 3.87 \\
\hline
\end{tabular}

$0.48 \mathrm{~cm}^{3}$ we performed the tests for 2400 time steps, on the time interval $[0,120] \mathrm{ms}$. The number of iterations remains bounded and almost constant during the test, as shown in Figures 3 and 4 . We notice a huge difference between the linear iteration of multigrid and the dual-primal preconditioners, with a reduction of more than $90 \%$ for the latter. If we focus on the trend of the dual-primal preconditioners' average number of linear iterations (Figures 3 and 4, right), we see that on both domains FETI-DP is affected by the different phases of the action potential: there is an initial peak during the activation phase, followed by an increase in the number of linear iterations as the potential propagates in the tissue and by a slow decrease as wider portions of tissue return to resting. We observe a better performance of the dual-primal preconditioners in terms of average CPU time per time step (see Table 3).
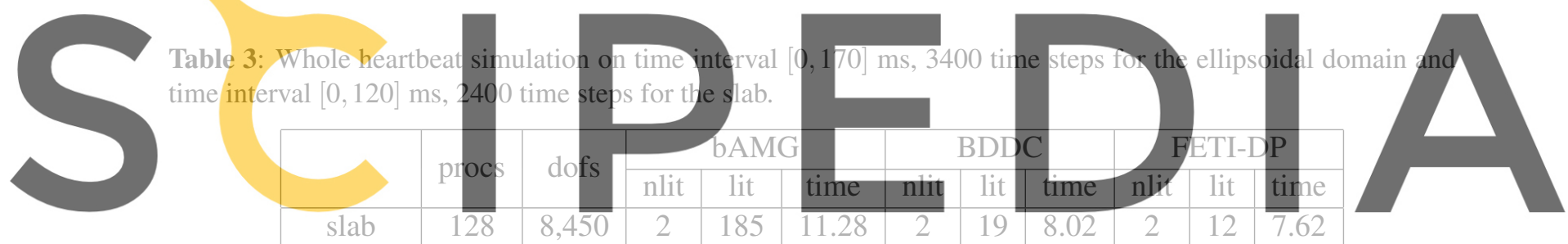

Register for free at https//WWW.scipedia.com to download the version without the watermark

\section{Conclusions}

We have constructed dual-primal preconditioners for fully implicit discretizations of the Bidomain system, which are solved through a decoupling strategy. A convergence bound for the preconditioned operator with deluxe scaling is validated numerically through extensive parallel numerical tests; efficiency and robustness of the solver is shown, thus enlarging the class of methods available for the efficient and accurate numerical solution of this complex cardiac reaction-diffusion model.

\section{REFERENCES}

[1] S. Balay et al., PETSc web page, https://www.mcs.anl.gov/petsc/ (2019).

[2] H. Chen, X. Li and Y. Wang, A two-parameter modified splitting preconditioner for the Bidomain equations, Calcolo, 56-2, 21 (2019).

[3] P. Colli Franzone, L.F. Pavarino and S. Scacchi, A numerical study of scalable cardiac electromechanical solvers on HPC architectures, Front. Physiol., 9-268 (2018). 

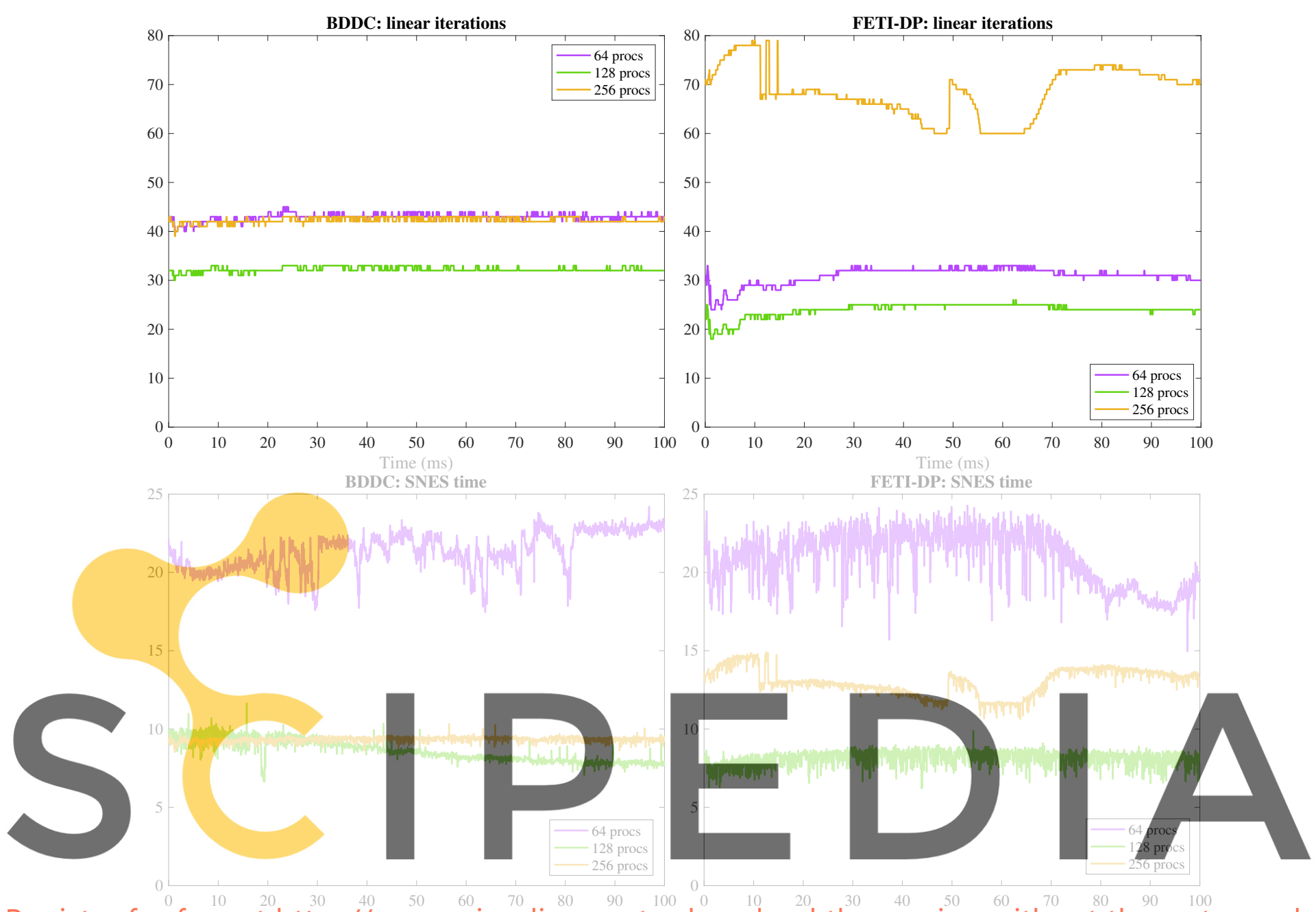

Register for free at https//wwwsscipedia.com to download the version without the watermark

Figure 1: Ellipsoidal domain. Strong scaling test over a time interval of $[0,100] \mathrm{ms}, 2000$ time steps. Fixed global mesh of $192 \cdot 96 \cdot 24$ elements $(936,050$ dofs), increasing number of processors. Comparison between BDDC (left column) and FETI-DP (right column) preconditioners. Top: average number of linear iterations per time step; bottom: average CPU time in seconds of each SNES solver call.

[4] P. Colli Franzone and G. Savaré, Degenerate evolution systems modeling the cardiac electric field at micro-and macroscopic level, in Evolution equations, semigroups and functional analysis, Springer (2002), pp. 49-78.

[5] P. Colli Franzone, L.F. Pavarino and S. Scacchi, Mathematical cardiac electrophysiology, Springer (2014).

[6] C.R. Dohrmann, A preconditioner for substructuring based on constrained energy minimization, SIAM J. Sci. Comput., 25-1 (2003), pp. 246-258.

[7] C.R. Dohrmann, O.B. Widlund, A BDDC algorithm with deluxe scaling for three-dimensional $H$ (curl) problems, Commun. Pure Appl. Math., 69-4 (2016), pp. 745-770. 

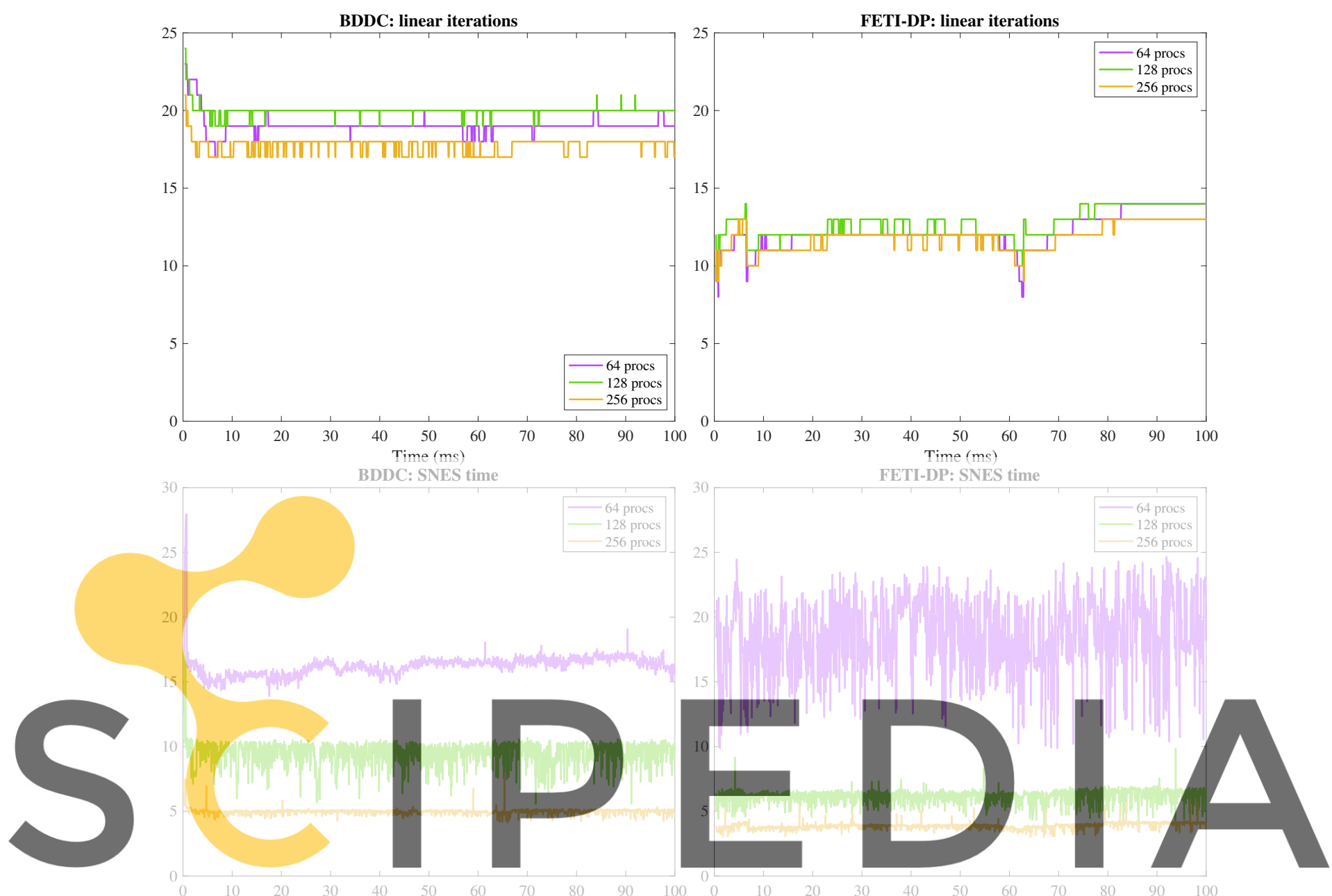

Register for free at https//www.scipedia.com to download the version without the watermark

Figure 2: Slab domain. Strong scaling test over a time interval of $[0,100] \mathrm{ms}, 2000$ time steps. Fixed global mesh of 192 $96 \cdot 24$ elements (936,050 dofs), increasing number of processors. Comparison between BDDC (left column) and FETI-DP (right column) preconditioners. Top: average number of linear iterations per time step; bottom: average CPU time in seconds of each SNES solver call.

[8] C. Farhat, M. Lesoinne, P. LeTallec, K. Pierson and D. Rixen, FETI-DP: a dual-primal unified FETI method-part I: A faster alternative to the two-level FETI method, Int. J. Numer. Methods Engrg., 50-7 (2001), pp. 1523-1544.

[9] I.J. LeGrice, B.H. Smaill, L.Z. Chai, S.G. Edgar, J.B. Gavin and P.J. Hunter, Laminar structure of the heart: ventricular myocyte arrangement and connective tissue architecture in the dog, Amer. J. Physiol.-Heart Circ.Physiol., 269-2 (1995), pp. H571-H582.

[10] P. LeTallec, J. Mandel and M. Vidrascu, A Neumann-Neumann domain decomposition algorithm for solving plate and shell problems, SIAM J. Numer. Anal., 35-2 (1998), pp. 836-867.

[11] J. Li and O.B. Widlund, FETI-DP, BDDC, and block Cholesky methods, Internat. J. Numer. Meth- 

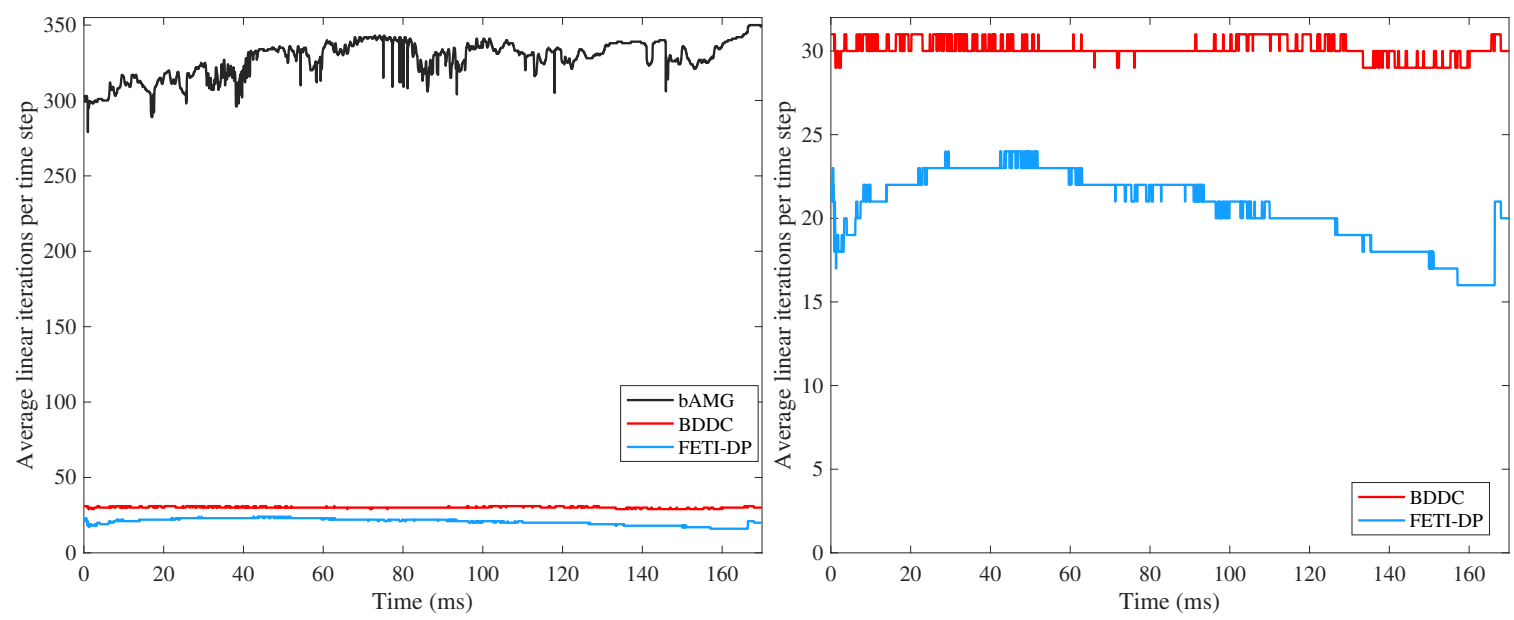

Figure 3: Whole heartbeat simulation on ellipsoidal domain, time interval $[0,170] \mathrm{ms}, 3400$ time steps. Average number of linear iterations per time step (left), zoom over dual-primal preconditioner (right).
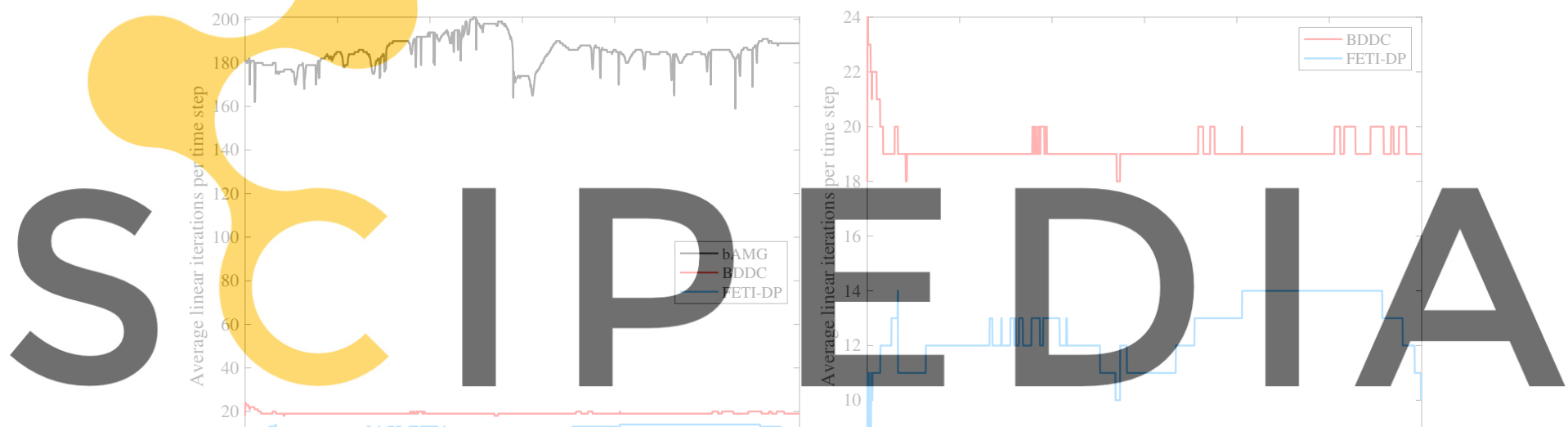

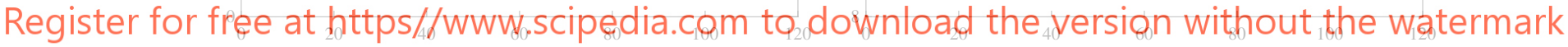
Time (ms)

Time (ms)

Figure 4: Whole heartbeat simulation on slab domain, time interval $[0,120] \mathrm{ms}, 2400$ time steps. Average number of linear iterations per time step (left), zoom over dual-primal preconditioner (right).

ods Engrg., 66-2 (2006), pp. 250-271.

[12] C. Luo and Y. Rudy, A model of the ventricular cardiac action potential. Depolarization, repolarization, and their interaction., Circ. Res., 68-6 (1991), pp. 1501-1526.

[13] M. Munteanu and L.F. Pavarino, Decoupled Schwarz algorithms for implicit discretizations of nonlinear Monodomain and Bidomain systems, Math. Models Methods Appl. Sci., 19-7 (2009), pp. 1065-1097.

[14] M. Munteanu, L.F. Pavarino and S. Scacchi, A scalable Newton-Krylov-Schwarz method for the Bidomain reaction-diffusion system, SIAM J.Sci. Comput., 31-5 (2009), pp. 3861-3883.

[15] M. Murillo and X-C. Cai, A fully implicit parallel algorithm for simulating the non-linear electri- 
cal activity of the heart, Numer. Linear Algebra Appl., 11 (2004), pp. 261-277.

[16] L.F. Pavarino, S. Scacchi, S. Zampini, Newton-Krylov-BDDC solvers for nonlinear cardiac mechanics, Comput. Methods Appl. Mech. Engrg, 295 (2015), pp. 562-580.

[17] M. Pennacchio, G. Savaré and P. Colli Franzone, Multiscale modeling for the bioelectric activity of the heart, SIAM J. Math. Anal., 37-4 (2005), pp. 1333-1370.

[18] A. Quarteroni, A. Manzoni and C. Vergara, The cardiovascular system: mathematical modelling, numerical algorithms and clinical applications, Acta Numer., 26 (2017), pp. 365-590.

[19] A. Quarteroni, T. Lassila, S. Rossi and R. Ruiz-Baier, Integrated Heart - Coupling multiscale and multiphysics models for the simulation of the cardiac function, Comput. Methods Appl. Mech. Engrg, 314 (2017), pp. 345-407.

[20] J.M. Rogers and A.D. McCulloch, A collocation-Galerkin finite element model of cardiac action potential propagation, IEEE Trans. Biomed. Engrg., 41-8 (1994), pp. 743-757.

[21] S. Scacchi, A multilevel hybrid Newton-Krylov-Schwarz method for the Bidomain model of electrocardiology, Comput. Methods Appl. Mech. Engrg, 200 (2011), pp. 717-725.

[22] J. Sundnes, G.T. Lines and A. Tveito, An operator splitting method for solving the bidomain equations coupled to a volume conductor model for the torso, Math.Biosci., 194-2 (2005), pp. 233-248.

[23] K.H.W.J. Ten Tusscher, D. Noble, P-J. Noble and A.V. Panfilov, A model for human ventricular tissue, Amer. J. Physiol.-Heart Circ. Physiol., 286-4 (2004), pp. H1573-H1589.

[24] A. Toselli and O. Widlund, Domain decomposition methods-algorithms and theory, Springer (2006).

[25] S. Zampini, Dual-primal methods for the cardiac Bidomain model, Math. Models Methods Appl. Sci., 24-4 (2014), pp. 667-696. 